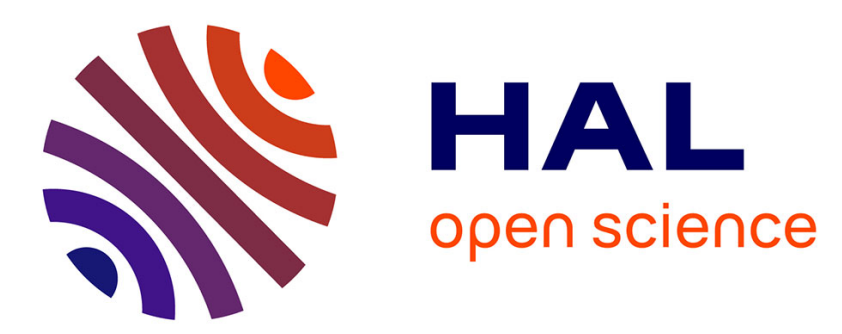

\title{
Optimal Control of Plant Disease Epidemics with Clean Seed Usage
}

Frédéric Marie Hamelin, Brady Bowen, Pierre Bernhard, Vushrali A Bokil

\section{To cite this version:}

Frédéric Marie Hamelin, Brady Bowen, Pierre Bernhard, Vushrali A Bokil. Optimal Control of Plant Disease Epidemics with Clean Seed Usage. Bulletin of Mathematical Biology, 2021, 83 (5), pp.46. 10.1007/s11538-021-00872-w . hal-03200491

HAL Id: hal-03200491

https://institut-agro-rennes-angers.hal.science/hal-03200491

Submitted on 16 Apr 2021

HAL is a multi-disciplinary open access archive for the deposit and dissemination of scientific research documents, whether they are published or not. The documents may come from teaching and research institutions in France or abroad, or from public or private research centers.
L'archive ouverte pluridisciplinaire HAL, est destinée au dépôt et à la diffusion de documents scientifiques de niveau recherche, publiés ou non, émanant des établissements d'enseignement et de recherche français ou étrangers, des laboratoires publics ou privés. 


\title{
Optimal Control of Plant Disease Epidemics with Clean Seed Usage
}

\author{
Hamelin, F. M. • Bowen, B. • \\ Bernhard, P. • Bokil, V. A.
}

Received: date / Accepted: date

\begin{abstract}
The distribution and use of pathogen-free planting material ("clean seeds") is a promising method to control plant diseases in developing countries. We address the question of minimizing disease prevalence in plants through the optimal usage of clean seeds. We consider the simplest possible $S$ - $I$ model together with a simple economic criterion to be maximized. The static optimization problem shows a diversity of possible outcomes depending on economical and epidemiological parameters. We derive a simple condition showing to what extent subsidizing clean seeds relative to the epidemiological features of the disease may help eradicate or control the disease. Then we consider dynamic optimal control and Pontryagin's maximum principle to study the optimal usage of clean seeds to control the disease. The dynamical results are comparable to the static ones and are even simpler in some sense. In particular, the condition on the critical subsidy rate that makes clean seed usage economically viable is unchanged from the static optimization case. We discuss how these results may apply to the control of maize lethal necrosis (MLN) in East-Africa.
\end{abstract}

\section{Introduction}

Plant diseases cause economic devastation especially in developing countries by severely affecting production of staple food crops due to yield losses. Cassava

F. M. Hamelin

IGEPP, INRAE, Institut Agro, Univ Rennes, 35000, Rennes, France

E-mail: frederic.hamelin@agrocampus-ouest.fr

B. Bowen

Department of Mathematics, Oregon State University, Corvallis, OR 97331

E-mail: bowenbr@math.oregonstate.edu

P. Bernhard

Université Côte d'Azur, Inria, INRAE, CNRS, Sorbonne Université, Biocore, France

E-mail: Pierre.Bernhard@inria.fr

V. A. Bokil

Department of Mathematics, Oregon State University, Corvallis, OR 97331

E-mail: bokilv@math.oregonstate.edu 
mosaic disease (CMD) and Maize lethal necrosis (MLN) are some of the most damaging crop diseases in the world $[16,17]$.

CMD occurs in many regions across Africa, India, and Sri Lanka, areas in which cassava is considered a primary food crop [1]. As CMD significantly decreases tuber production, it is a major constraint to cassava production [5]. CMD is caused by several viruses such as the African cassava mosaic virus (ACMV). These viruses are vectored by the whitefly Bemisia tabaci. In addition, the virus propagates through cuttings from infected plants used for planting. Cassava is a vegetatively propagated crop, and infected plants may not be detected. The distribution of virus-free planting material is a promising method to control CMD [17].

MLN has recently emerged in sub-Saharan East Africa, Southeast Asia, and South America, with large impacts on smallholder farmers [16]. MLN is caused by coinfections between the Maize chlorotic mottle virus (MCMV) and a potyvirus such as the Sugarcane mosaic virus (SCMV). MCMV is spread by a variety of insect vectors including maize thrips Frankliniella williamsi. The symptoms of MLN include severe stunting and death, and productivity is all but lost in the stalks that are infected [12]. In addition to vector transmission of MCMV there is also a low, but epidemiologically significant, possibility of vertical transmission through seeds. The distribution and use of certified virus-free seeds is a promising method to control MLN [10].

From now on, we will use the term "clean seeds" to generically mean certified pathogen-free seeds, tubers, or in vitro propagated planting material [18]. The use of clean seeds can be combined with other control methods such as vector control, crop rotations, removal of infected plants ("roguing"), and breeding plants to be more resistant to pathogens.

Previous models $[14,10,15,19,2,9]$ computationally explored the effects of clean seeds in combination with other control methods with applications to MLN and CMD among other diseases. However, we are not aware of a generic mathematical model focusing on clean seeds as a control method. The article [5] addresses the effectiveness of several control methods in terms of virus evolution, with special attention to CMD. The authors note that in vitro propagation and cutting selection holds the risk of viral evolution resulting in an increased virus titre, while roguing does not carry the same degree of concern. The integration of epidemiological models of control with economic considerations is an active area of research in plant disease epidemiology $[4,7]$.

In this paper, we address the problem of optimally controlling plant diseases in crops using an epidemiological model and optimal control theory. Our model is a continuous time $S-I$ model based on ordinary differential equations in which we divide the host plant population into two compartments; susceptible to disease or healthy plant compartment, $S$, and infected plant compartment $I$. The model incorporates continual harvesting and replanting via a proportion of clean seeds. We do not consider insect vectors explicitly, instead considering them implicitly via horizontal transmission between plants. In addition, the virus can spread vertically via infected seeds.

We consider the question of how to control clean seed usage while optimizing revenue from harvesting of both healthy and infected plants. Towards this goal, we consider a profit functional where clean and infected plants are sold at different rates, along with the cost of replanting through the use of clean seeds. Both the healthy and infected plants are harvested though we assume that the infected 
plants are worth less per unit price than the healthy plants. Using the mathematical theory of optimal control we optimize over the revenue from harvesting by controlling the fraction of clean seeds that are used in planting.

An outline of the paper is as follows: We describe the epidemic model in Section 2, along with its biological interpretation. We then look into the static optimization of the system in Section 3 and determine a variety of cases that relate the use of clean seeds to epidemiological and economical parameters. We study the case of dynamic optimal control in Section 4, where we consider a finite end time with a full account of all remaining plants. Section 4 presents numerical simulations of the dynamical optimal control system. Finally, in Section 5, we present a discussion and conclusion of our results.

\section{$2 S-I$ Model of Plant Disease with Clean Seeds and Harvesting}

Our model is a modification of one presented in [5] where we include vertical plant-to-seed transmission in addition to removal of infected seeds, as well as seedto-plant transmission. The term "seed" here means planting material in general, including tubers and cuttings, depending on the crop considered.

The total density of the plant host is $N=S+I$, with $S$ and $I$ the densities of susceptible and infected hosts, respectively. We do not discriminate between young and adult plant hosts for simplicity. Hosts are harvested at rate $\mu$, and replanted at rate $\sigma$. The fraction of clean seeds is $\phi$. The probability that an infected plant produces infected seeds is $v$. The probability that infected seeds are detected and discarded is $p$. The fraction of infected plants contributing to local seed production is therefore $1-p v$. The probability that infected seeds produce infected plants is $q$. The disease transmission rate is denoted $\beta$. The equations for the $S$ and $I$ compartments are given by

$$
\begin{aligned}
\frac{\mathrm{d} S}{\mathrm{~d} t} & =\sigma \phi+\sigma(1-\phi) \frac{((1-v)+(1-q)(1-p) v) I+S}{S+I(1-p v)}-\mu S-\beta I S \\
\frac{\mathrm{d} I}{\mathrm{~d} t} & =\sigma(1-\phi) \frac{q(1-p) v I}{S+I(1-p v)}-\mu I+\beta I S
\end{aligned}
$$

Descriptions of the variables and parameters in the $S-I$ model are given in Table 1, with the default values used in our simulations and their corresponding ranges of values. Figure 1 displays a compartmental model for the dynamics involving replanting using seeds taken from the entire stock of plants, which corresponds to the fraction expressed in Equations (1).

To disentangle the use of clean seeds from related control methods such as seed selection, we then assume that growers are not able to detect between healthy and infected seeds $(p=0)$ and so both are used to propagate the host plant. Let $\nu=v q$ denote the vertical transmission rate of the pathogen. Equation (1) simplifies to

$$
\begin{aligned}
& \frac{\mathrm{d} S}{\mathrm{~d} t}=\sigma \phi+\sigma(1-\phi) \frac{(1-\nu) I+S}{N}-\mu S-\beta I S, \\
& \frac{\mathrm{d} I}{\mathrm{~d} t}=\sigma(1-\phi) \frac{\nu I}{N}-\mu I+\beta I S,
\end{aligned}
$$






Fig. 1 Replanting dynamics as expressed in Equation 1. The arrows represent the possible pathways that seeds can take from the compartments on the left to the compartments on the right. The parameters next to each arrow represent the probabilities that seeds move along that pathway.

\begin{tabular}{|l|l|l|c|}
\hline $\begin{array}{l}\text { Variables/ } \\
\text { Parameters }\end{array}$ & Description & $\begin{array}{l}\text { Range } \\
\text { of Values }\end{array}$ & $\begin{array}{c}\text { Default } \\
\text { Value }\end{array}$ \\
\hline$S$ & Susceptible plant population density & {$[0,1]$} & \\
$I$ & Infected plant population density & {$[0,1]$} & \\
$N$ & Total plant population density & 1 & \\
$\phi$ & Fraction of clean seeds used in replanting & {$[0,1]$} & \\
\hline$\sigma$ & Replanting rate & {$[0, \infty)$} & 1 \\
$\mu$ & Harvest rate & {$[0, \infty)$} & 1 \\
$\beta$ & Horizontal transmission rate & $\frac{1}{2}$ or $\frac{3}{2}$ \\
$v$ & Probability of plant-to-seed transmission & {$[0,1]$} & $\frac{2}{3}$ or 1 \\
$p$ & Probability of detection of infected seeds & {$[0,1]$} & 0 \\
$q$ & Probability of seed-to-plant transmission & {$[0,1]$} & 1 \\
\hline$p_{S}$ & Profit from selling uninfected plants & {$[0, \infty)$} & 3 \\
$p_{I}$ & Profit from selling infected plants & {$[0, \infty)$} & 0 \\
$c$ & Unit cost of using clean seeds & {$[0, \infty)$} & 1 \\
$P_{S}$ & End value of remaining uninfected plants & {$[0, \infty)$} & 0 or 2 \\
$P_{I}$ & End value of remaining infected plants & {$[0, \infty)$} & 0 \\
\hline
\end{tabular}

Table 1 List of variables and parameters in the $S-I$ model (Equation 1) and objective functional (Equation 3).

Our goal in this paper is to look at the optimal control problem with control variable $\phi(t)$, for which the objective functional

$$
J(\phi(\cdot))=P_{S} S(T)+P_{I} I(T)+\int_{0}^{T}\left[p_{S} \mu S(t)+p_{I} \mu I(t)-c \sigma \phi(t)\right] \mathrm{d} t,
$$

is maximized. Thus, our objective is to maximize revenue by controlling the introduction of clean seeds. The objective functional represents the total profit from the patch of crops; it contains the values of the healthy and infected plants at the final time, the selling of both healthy and infected crops over the season, and the cost of replenishing harvested crops through clean seeds. More specifically, the parameter $c$ represents an additional cost of clean seeds relative to local seeds. The costs that do not depend on clean seed usage are left implicit in this functional. 
In the sequel, we will consider two subcases, 1) $P_{S}=P_{I}$ (we disregard the disease prevalence in the end), and 2) $P_{S}>P_{I}$ (we value disease prevalence in the end).

For simplicity, we assume that each harvested plant is replaced by the replanting of a new plant (resulting in $\sigma=\mu N$ ), which is a reasonable assumption in agriculture. As a consequence, $N$ is a constant from the equation for the total plant density,

$$
\frac{\mathrm{d} N}{\mathrm{~d} t}=\sigma-\mu N=0 .
$$

Without loss of generality, we can assume that $N=1$, and thus we have $\mu=\sigma$. Then the disease prevalence is $I=1-S$, and the state equations and the optimal control problem simplify to maximizing the functional

$$
J(\phi(\cdot))=P_{S} S(T)+P_{I}(1-S(T))+\int_{0}^{T}\left[p_{S} \mu S(t)+p_{I} \mu(1-S(t))-c \mu \phi(t)\right] \mathrm{d} t,
$$

subject to the state equation (2a), which with $\sigma=\mu$ simplifies to the equation

$$
\frac{\mathrm{d} S}{\mathrm{~d} t}=(\mu \phi \nu-\beta S+\mu(1-\nu))(1-S)
$$

We can rewrite Equation (4) in the form

$$
J(\phi(\cdot))=\left(P_{S}-P_{I}\right) S(T)+\left(P_{I}+T \mu p_{I}\right)+\int_{0}^{T}\left[\left(p_{S}-p_{I}\right) \mu S(t)-c \mu \phi(t)\right] \mathrm{d} t .
$$

In this form, we determine that maximizing this objective functional is the same as maximizing the functional (keeping the same notation for convenience)

$$
J(\phi(\cdot))=\left(P_{S}-P_{I}\right) S(T)+\int_{0}^{T}\left[\left(p_{S}-p_{I}\right) \mu S(t)-c \mu \phi(t)\right] \mathrm{d} t .
$$

Without loss of generality, we assume that both $p_{I}=0$ and $P_{I}=0$. In the case where either of these quantities is not zero, we can instead consider $P_{S}$ and $p_{S}$ to be the difference between the value of clean plants and the value of infected plants, for the cases of terminal payoff and running payoff respectively.

Thus, incorporating all our assumptions and simplifications, the problem that we will consider in the next sections, based on equations (5) and (6), is the maximization problem

$$
\begin{aligned}
& \max _{\phi} J(\phi(\cdot)):=P_{S} S(T)+\int_{0}^{T}\left[\mu\left(p_{S} S(t)-c \phi(t)\right)\right] \mathrm{d} t \\
& \text { subject to } \frac{\mathrm{d} S}{\mathrm{~d} t}=(\mu \phi \nu-\beta S+\mu(1-\nu))(1-S), S(0)=S_{0}, \\
& 0 \leq \phi(t) \leq 1 .
\end{aligned}
$$

We address this dynamic optimal control problem in Section 4. However, in the next section, we first consider a static optimization problem. 
3 Maximizing the long-term running payoff

In this section, we are interested in finding the optimal (constant) control $\phi$ that maximizes the long-term running payoff

$$
\bar{\ell}(\phi):=p_{S} \bar{S}(\phi)-c \phi,
$$

in which $\bar{S}(\phi)$ is the equilibrium value asymptotically reached by the state ODE

$$
\frac{\mathrm{d} S}{\mathrm{~d} t}=(\mu \phi \nu-\beta S+\mu(1-\nu))(1-S) .
$$

Thus, our maximization problem of interest here is to find

$$
\begin{aligned}
& \phi^{*}=\underset{\phi}{\arg \max } \bar{\ell}(\phi), \\
& \text { Subject to }(\mu \phi \nu-\beta S+\mu(1-\nu))(1-S)=0, \\
& \quad 0 \leq \phi \leq 1 .
\end{aligned}
$$

3.1 The Basic Reproduction Number for the Epidemic Model

We first define an important threshold parameter for the state ODE (9), that will aid in considering the relative importance of the transmission rate to the harvesting rate. The parameter $R$ is defined as

$$
R=\frac{\beta}{\mu}
$$

The basic reproduction number for the epidemic model (9) can be expressed as

$$
\mathcal{R}_{0}=\underbrace{(1-\phi) \nu}_{\text {vertical transmission }}+\underbrace{R}_{\text {horizontal transmission }},
$$

and can be interpreted as the sum of two different modes of transmission of the disease; vertical and horizontal transmission. There are two equilibrium values of $S$ :

1. (DFE) $S=1$, the Disease Free Equilibrium

2. (EE) $S=\bar{S}$, the Endemic Equilibrium, with

$$
\begin{aligned}
\bar{S} & :=\frac{\mu}{\beta}(\phi \nu+(1-\nu)), \\
& =1+\frac{1-\mathcal{R}_{0}}{R} .
\end{aligned}
$$

The DFE and EE are reached depending on whether $\mathcal{R}_{0}<1$ or $\mathcal{R}_{0}>1$, respectively. Using the DFE and the EE, we can rewrite the state equation (9) as

$$
\frac{\mathrm{d} S}{\mathrm{~d} t}=\beta(\bar{S}-S)(1-S)
$$

The EE, $\bar{S}$, exists and is biologically feasible when $\mathcal{R}_{0}>1$. In this case, the equation for $S$ indicates that $\bar{S}$ is locally asymptotically stable, while $S=1$ is unstable. When $\mathcal{R}_{0}<1$, the $\operatorname{DFE}(S=1)$ is the only equilibrium that is biologically feasible and is stable. 
Remark 1 In the absence of control $(\phi=0), \mathcal{R}_{0}$ equals $R+\nu$. If $R+\nu<1$, the disease gets extinct in the absence of control (which is costly to implement). Then the optimal strategy is trivially $\phi^{\star}=0$. Therefore, from now on, we make the following assumption:

(A1): $R+\nu>1$.

3.2 Dynamics of the epidemic model

In this section, we analyze the $S-I$ model for its equilibria. We first define a second threshold quantity

$$
\bar{\phi}:=1-\frac{1-R}{\nu}
$$

The threshold $\bar{\phi}$, as a function of the parameters $R$ and $\nu$, is the value of $\phi$ at which $\mathcal{R}_{0}=1$. Using the threshold $\bar{\phi}$, we can rewrite the basic reproduction number, given in equation (14) as

$$
\mathcal{R}_{0}=1+\nu(\bar{\phi}-\phi)
$$

We also rewrite the EE in equation (15) in terms of the threshold $\bar{\phi}$ as

$$
\begin{aligned}
\bar{S} & :=\phi \frac{\nu}{R}+\frac{(1-\nu)}{R}, \\
& =1-\frac{\nu(\bar{\phi}-\phi)}{R}
\end{aligned}
$$

In the analysis of the $S-I$ model, two cases are to be distinguished:

(A) $R>1$ : In this case, the threshold $\bar{\phi}>1$. The equation for $S$ asymptotically reaches the endemic equilibrium (EE), $\bar{S}$, for all $\phi \in[0,1]$. In particular, for full control $\phi=1$, we have that the equilibrium is $\bar{S}=\mu / \beta=1 / \mathcal{R}_{0}=1 / R<$ 1. This means that the prevalence of the disease is $\bar{I}=1-1 / R>0$, and one cannot get rid of the disease.

(B) $R<1$ : In this case, the threshold $\bar{\phi}<1$, and $S$ attains the endemic equilibrium, $\bar{S}$, only on the interval $\phi \in[0, \bar{\phi})$. For $\phi \in[\bar{\phi}, 1], S$ approaches the disease free equilibrium (DFE), $S=1$. This means that the disease approaches extinction and that is a sufficient rate of control to get rid of the disease.

In either case, the absence of control $(\phi=0)$ results in the endemic equilibrium $\bar{S}=(1-\nu) / R$. This means that the pathogen has a positive equilibrium for any vertical transmission $\nu$ and will invade the entire plant population when there is full vertical transmission, i.e. when $\nu=1$. These results are collectively depicted in Figure 2. 

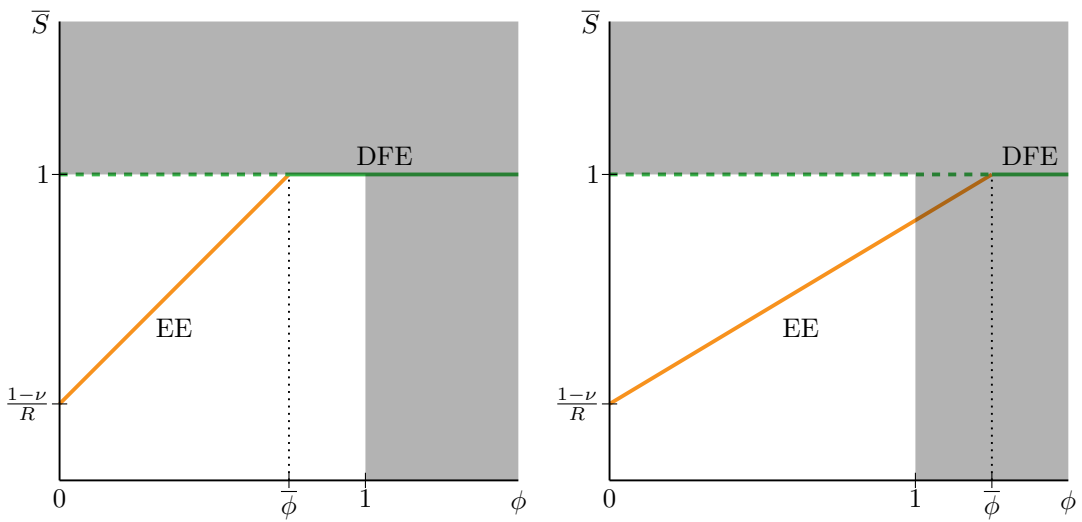

Fig. 2 Dynamics of the $S-I$ disease model. $\bar{\phi}=1-\frac{1-R}{\nu}$. DFE means Disease free equilibrium, $\mathrm{EE}$ means endemic equilibrium. The biologically feasible region is in white. (Left) Case (B): Dynamics for the case $R<1$. (Right) Case (A): Dynamics for the case $R>1$.

\subsection{Static optimization}

In this section, we consider static optimization of the problem. In this case, the relative unit price of healthy plants, $p_{S}$, versus clean seeds, $c$, also becomes important. We denote the ratio of the two parameters, $p_{S}$ and $c$, as a third threshold quantity $r$

$$
r=\frac{p_{S}}{c} .
$$

We start with distinguishing the same two cases as in the dynamics of the $S-I$ model.

(A) $R>1$. Then, $\bar{\phi}>1$, and for all $\phi \in[0,1], S$ asymptotically reaches the EE equilibrium. We have

$$
\begin{aligned}
\bar{\ell}(\phi) & :=p_{S} \bar{S}(\phi)-c \phi=p_{S} \frac{\mu}{\beta}(\phi \nu+(1-\nu))-c \phi \\
& =\phi c\left(\frac{\nu r}{R}-1\right)+c r \frac{(1-\nu)}{R} \\
& =c\left[r-\phi-\frac{\nu r}{R}(\bar{\phi}-\phi)\right],
\end{aligned}
$$

Two sub-cases are then to be distinguished:

(A1) $R<\nu r$. Then $\phi^{\star}=1$.

(A2) $R>\nu r$. Then $\phi^{\star}=0$.

(B) $R<1$. Then, $\bar{\phi}<1$, and $S$ asymptotically reaches the EE on $[0, \bar{\phi})$ and the DFE on $[\bar{\phi}, 1]$. Thus, we have

$$
\bar{\ell}(\phi)= \begin{cases}\phi c\left(\frac{\nu r}{R}-1\right)+c r \frac{(1-\nu)}{R}=c\left[r-\phi-\frac{\nu r}{R}(\bar{\phi}-\phi)\right] & \text { for } \phi \in[0, \bar{\phi}) \\ c(r-\phi) & \text { for } \phi \in[\bar{\phi}, 1]\end{cases}
$$


We note that $\bar{\ell}(\phi)$ is continuous over $[0,1]$. The derivative of $\bar{\ell}$ with respect to $\phi$ is:

$$
\bar{\ell}^{\prime}(\phi)= \begin{cases}c\left(\frac{\nu r}{R}-1\right) & \text { for } \phi \in(0, \bar{\phi}) \\ -c<0 & \text { for } \phi \in(\bar{\phi}, 1)\end{cases}
$$

Note that $\bar{\ell}^{\prime}(\phi)$ has a discontinuity at $\phi=\bar{\phi}$. Two sub-cases are then to be distinguished:

(B1) If $R<\nu r$, then $\bar{\ell}^{\prime}(\phi)>0$ for $\phi \in(0, \bar{\phi})$ and $\bar{\ell}^{\prime}(\phi)<0$ for $\phi \in(\bar{\phi}, 1)$. Therefore, $\phi^{\star}=\bar{\phi}$.

(B2) Otherwise if $R>\nu r$ then $\bar{\ell}^{\prime}(\phi)<0$ for $\phi \in(0,1)$. Therefore, $\phi^{\star}=0$.
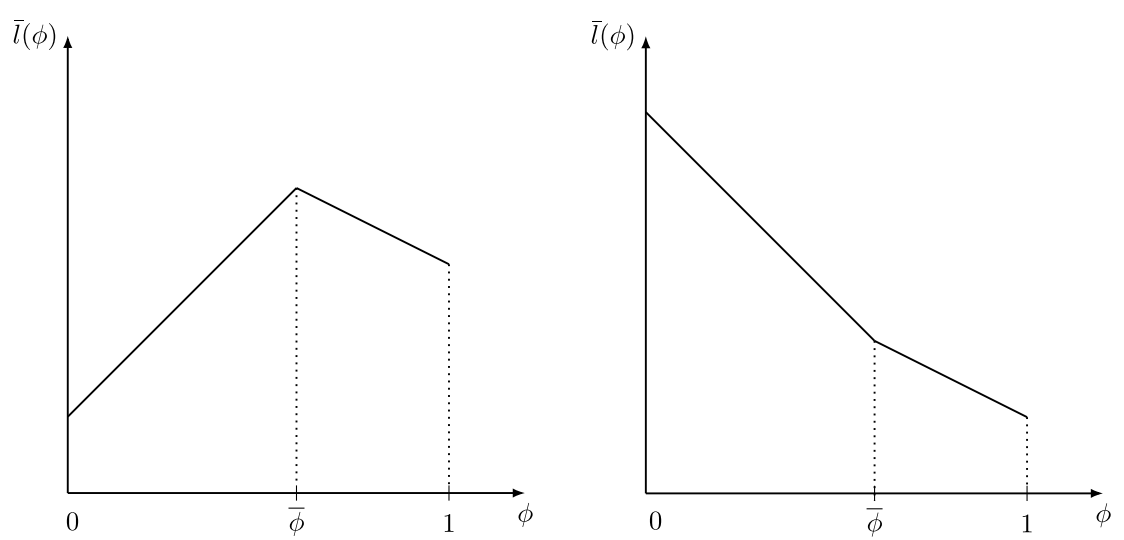

Fig. 3 Long term payoff as a function of $\phi$ in the case (B) $R<1$. (Left) Case B1: the maximum occurs at $\phi=\bar{\phi}$. (Right) Case B2: the maximum occurs at $\phi=0$.

\subsection{Discussion of Static Optimization Results}

The results from static optimization can be summarized in the parameter plane $(R, r)$ as below and in Figure 4.

(S1) If $R<1-\nu$, (i.e., assumption (A1) as stated in Remark 1 does not hold), then $\phi^{\star}=0$, and the system converges to DFE $S=1$.

(S2) If $R>1-\nu$ (assumption (A1) holds) and $R>\nu r$, then $\phi^{\star}=0$ again, but the system converges to EE $\bar{S}=(1-\nu) / R$.

(S3) If $R<\nu r$ and $R>1$, then $\phi^{\star}=1$, and the system converges to EE $\bar{S}=1 / R$.

(S4) If $R<\nu r$ and $1-\nu<R<1$, then $\phi^{\star}=\bar{\phi}$ and the system converges to DFE $S=1$.

Figure 4 displays this summary by dividing the $(R, r)$ plane into distinct regions of differing control strategies. Biologically we may interpret the results from static optimization as follows: 


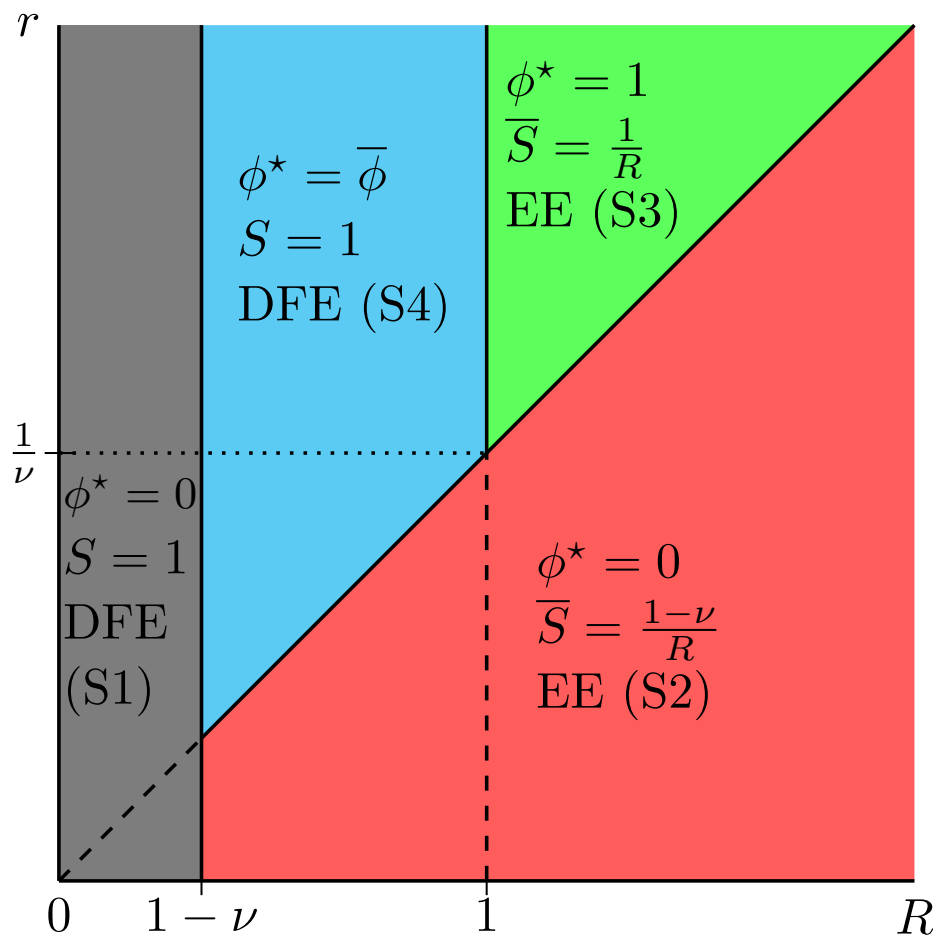

Fig. 4 Results of static optimization: The $(R, r)$ plane is divided into distinct regions with differing control strategies.

(S1) If $R<1-\nu$, then $\mathcal{R}_{0}<1$ for $\phi=0$ and the disease goes extinct in absence of control (which is costly to implement). Therefore, there is no incentive to control, and $\phi^{\star}=0$.

(S2) If $R>1-\nu$ and $R>\nu r$, then the disease transmission rate $(\beta$ in $R=\beta / \mu$ ) is too large relative to the plant renewal rate $(\mu)$ or to vertical transmission $(\nu)$, to make it worthy to use clean seeds (which have a relative cost $1 / r$ ). Hence $\phi^{\star}=0$, meaning that the disease is left uncontrolled and stabilizes at an endemic equilibrium.

(S3) If $R<\nu r$ and $R>1$, then the optimal strategy is to use as many clean seeds as possible $\left(\phi^{\star}=1\right)$ to minimize the prevalence of the disease, which remains at an endemic equilibrium.

(S4) If $R<\nu r$ and $1-\nu<R<1$, then it is possible to get rid of the disease by using an intermediate proportion of clean seeds (any $\phi \in[\bar{\phi}, 1]$ ). Since clean seeds are costly, the optimal proportion of clean seeds is $\phi^{\star}=\bar{\phi}$, and the disease goes extinct.

\subsubsection{Decreasing the cost of clean seeds}

Case (S2) is discouraging. One may wonder whether subsidizing clean seeds may help with controlling the disease. Let $s$ be the discount on the unit price of clean 
seeds due to subsidies, i.e. the unit price of clean seeds is now $c(1-s)$ instead of $c$. Thus, $r=p_{S} / c$ is now replaced with $r /(1-s)$, and the condition $R<\nu r$ becomes

$$
R<\frac{\nu r}{1-s}
$$

Therefore, if the discount due to subsidies on clean seeds exceeds a critical fraction, i.e.,

$$
s>1-\frac{\nu r}{R},
$$

then Case (S2) can be avoided and the outcome of static optimization will be determined by either Case (S3) or Case (S4).

\subsubsection{Decreasing vertical transmission}

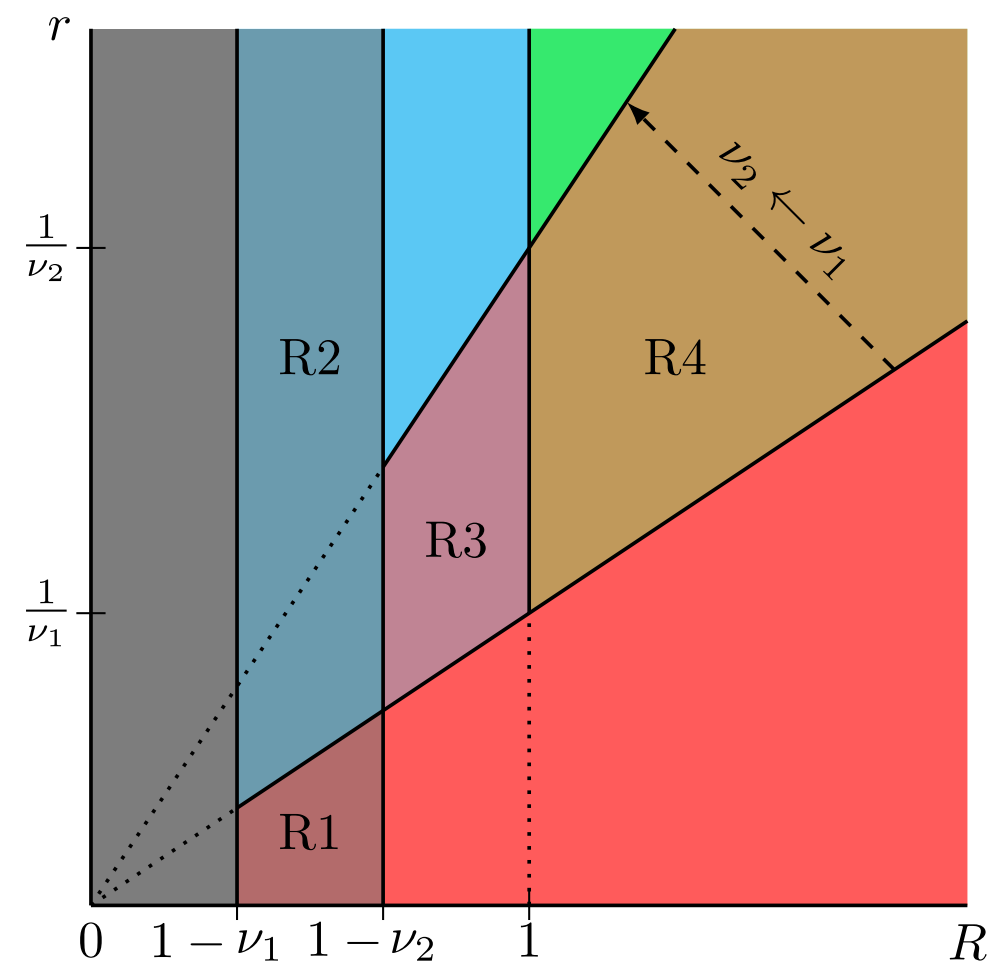

Fig. 5 Changes to the $(R, r)$ plane as $\nu$ varies: Decreasing the value of $\nu$ from $\nu_{1}$ to $\nu_{2}$ results in 4 regions where the control strategy has changed.

Figure 5 shows the effect of decreasing vertical transmission $\nu$ in the parameters plane $(R, r)$. Decreasing $\nu$ has a positive effect in regions $\mathrm{R} 1$ and $\mathrm{R} 2$. In region $\mathrm{R} 1$, the system switches from an uncontrolled $\left(\phi^{\star}=0\right)$ endemic equilibrium (EE) to disease-free equilibrium (DFE) requiring no control $\left(\phi^{\star}=0\right)$. In region $\mathrm{R} 2$, the system switches from controlled $\left(\phi^{\star}=\bar{\phi}\right)$ DFE to DFE with no control $\left(\phi^{\star}=0\right)$. 
By contrast, decreasing vertical transmission $(\nu)$ has a negative effect in regions R3 and R4. In region R3, the system switches from controlled $\left(\phi^{\star}=\bar{\phi}\right)$ DFE to uncontrolled $\left(\phi^{\star}=0\right)$ EE. In region R4, the system switches from controlled $\left(\phi^{\star}=1\right)$ EE $(\bar{S}=1 / R)$ to uncontrolled $\left(\phi^{\star}=0\right)$ EE $(\bar{S}=(1-\nu) / R)$. Note that even though the decrease in $\nu$ has a direct positive effect as it decreases the disease prevalence for a given $\phi$, the net resulting effect is negative, as $\phi^{\star}$ switches from 1 to 0 (meaning that it is no longer optimal to use clean seeds).

\section{Dynamic Optimal Control}

In this section, we address the dynamic optimal control problem stated in Section 2 in System (7). Based on the objective functional, $J(\phi(\cdot))$ and the state ODE and constraints in System (7) we derive the following Hamiltonian:

$$
\mathcal{H}=\mu\left[p_{S} S(t)-c \phi(t)\right]+\lambda(t)[(\nu \mu \phi(t)-\beta S(t)+\mu(1-\nu))(1-S(t))] .
$$

This gives us the following adjoint equation:

$$
\frac{\mathrm{d} \lambda}{\mathrm{d} t}=-\frac{\partial \mathcal{H}}{\partial S}=-\mu p_{S}+\lambda(t)[(1-S(t)) \beta+(\nu \mu \phi(t)-\beta S(t)+\mu(1-\nu))] .
$$

Since the Hamiltonian is linear in $\phi$, the optimality condition will contain no information on the control and we cannot use this to find a characterization of the optimal control. In Appendix C, Section 8 we show that if $R \neq r \nu$, then this problem has a bang-bang optimal control, that is the optimal control $\phi^{\star}$ cannot be singular on an interval.

We define the switching function

$$
\psi(t)=\frac{\partial \mathcal{H}}{\partial \phi}=\mu(-c+\lambda(t)(1-S(t)) \nu),
$$

Using the switching function, we characterize the optimal control as

$$
\phi^{\star}(t)=\left\{\begin{array}{ll}
0 & \text { if } \psi(t) \leq 0 \\
1 & \text { if } \psi(t)>0
\end{array} .\right.
$$

According to Pontryagin's maximum principle, the transversality condition is

$$
\lambda(T)=P_{S} .
$$

Next, we solve the state and adjoint equations in backward time from every possible value of $S(T)$. This will yield a field of optimal trajectories in the plane $(t, S)$ (in blue in Fig. 6). This method will allow us to derive a switching curve in the plane $(t, S)$ (in red in Fig. 6). Getting back to the original problem (7), the optimal strategy is the one starting from $\left(t=0, S=S_{0}\right)$.

Defining $S_{T}=S(T)$,

$$
\psi(T)=-\mu c+P_{S}\left(1-S_{T}\right) \nu \mu .
$$

We define a threshold value for $S_{T}$ as

$$
\hat{S}_{T}=1-\frac{c}{\nu P_{S}} .
$$


If $S_{T}>\hat{S}_{T}$, then $\psi(T)<0$ and $\phi^{\star}(T)=0$. Otherwise if $S_{T}<\hat{S}_{T}$, (which implies $\hat{S}_{T}>0$ or equivalently $\left.\nu P_{S}>c\right), \psi(T)>0$ and $\phi^{\star}(T)=1$.

From now on, we focus on the $\phi^{\star}(T)=0$ case to derive a switching curve in the $(t, S)$ plane.

As long as $\phi=0$ (in backward time), we have, introducing a dot to denote differentiation w.r.t. time,

$$
\begin{aligned}
& \dot{S}=(1-S)(-\beta S+\mu(1-\nu)), \\
& \dot{\lambda}=\lambda(\beta(1-2 S)+\mu(1-\nu))-\mu p_{S}, \quad \lambda(T)=P_{S},
\end{aligned}
$$

and

$$
\psi=\mu[\nu \lambda(1-S)-c] \Longleftrightarrow \nu \lambda(1-S)=\frac{\psi}{\mu}+c .
$$

This yields

$$
\begin{aligned}
\dot{\psi} & =\nu \mu[\dot{\lambda}(1-S)-\dot{S} \lambda], \\
& =\nu \mu(1-S)\left[\lambda(\beta(1-2 S)+\mu(1-\nu))-\mu p_{S}-\lambda(\mu(1-\nu)-\beta S)\right], \\
& =\nu \mu(1-S)\left[\lambda \beta(1-S)-\mu p_{S}\right], \\
& =\nu \mu(1-S)\left[\frac{\beta}{\nu}\left(\frac{\psi}{\mu}+c\right)-\mu p_{S}\right], \\
& =\mu(1-S)\left[\frac{\beta}{\mu} \psi+\beta c-\mu \nu p_{S}\right],
\end{aligned}
$$

with $\psi(T)=\mu\left[\nu P_{S}\left(1-S_{T}\right)-c\right]<0$ (since we assume $\phi^{\star}(T)=0$ ). Using the expression of $\hat{S}_{T}$ in (25), one may notice that for all $S_{T}>\hat{S}_{T}$,

$$
\dot{\psi}(T)=\nu \mu\left(1-S_{T}\right)\left(\beta P_{S}\left(1-S_{T}\right)-\mu p_{S}\right)<0
$$

if and only if $R / \nu<r$.

Let $t^{\star}$ be a switching time such that $\psi\left(t^{\star}\right)=0$. Then

$$
\dot{\psi}\left(t^{\star}\right)=\mu\left(1-S\left(t^{\star}\right)\right)\left[\beta c-\mu \nu p_{S}\right] .
$$

Consequently, switching from $\phi=0$ to $\phi=1$ (in backward time) is possible iff

$$
\beta c-\mu \nu p_{S}<0 \Longleftrightarrow \frac{R}{\nu}<r .
$$

(The adjoint and state variables are necessarily continuous, so the switching function $\psi$ is continuous as well.) As a corollary, the condition $R / \nu>r$ implies that the switching function $\psi$ never crosses zero and so the optimal control is $\phi^{\star}=0$ for all $t<T$.

If $R / \nu<r$, then $\dot{\psi}<0$ (since $\psi(T)<0$ ) as long as $\phi=0$ (in backward time).

Since the Hamiltonian is constant all along the optimal trajectory (see appendix A), we have, for all $t$ such that $\phi^{\star}=0$ :

$$
\begin{aligned}
H(T) & =H(t), \\
\mu p_{S} S_{T}+P_{S}\left(\mu(1-\nu)-\beta S_{T}\right)\left(1-S_{T}\right) & =\mu p_{S} S+\lambda(\mu(1-\nu)-\beta S)(1-S),
\end{aligned}
$$


which yields

$$
\lambda(t)=\frac{\mu p_{S}\left(S(t)-S_{T}\right)+P_{S}\left(1-S_{T}\right)\left(\beta S_{T}-\mu(1-\nu)\right)}{(\beta S(t)-(1-\nu) \mu)(1-S(t))} .
$$

Thus,

$$
\psi(t)=\mu\left[\frac{\nu\left(\mu p_{S}\left(S(t)-S_{T}\right)+P_{S}\left(1-S_{T}\right)\left(\beta S_{T}-\mu(1-\nu)\right)\right)}{\beta S(t)-(1-\nu) \mu}-c\right] .
$$

From this we get

$$
S\left(t^{\star}\right)=\frac{c \mu+\nu\left(P_{S} \beta S_{T}\left(1-S_{T}\right)-\mu\left((1-\nu)\left(1-S_{T}\right) P_{S}+S_{T} p_{S}+c\right)\right)}{\beta c-\nu \mu p_{S}} .
$$

Solving $\dot{S}=(1-S)(-\beta S+\mu(1-\nu))$ with terminal condition $S(T)=S_{T}$, we get for all $t \in\left[t^{\star}, T\right]$ :

$$
S(t)=\frac{\left(\beta S_{T}-\mu(1-\nu)\right) \exp [(\beta-\mu(1-\nu))(T-t)]+\mu(1-\nu)\left(1-S_{T}\right)}{\left(\beta S_{T}-\mu(1-\nu)\right) \exp [(\beta-\mu(1-\nu))(T-t)]+\beta\left(1-S_{T}\right)} .
$$

Equating the expressions of $S\left(t^{\star}\right)$ given by Equations (26) and (27) yields at most 3 possible solutions for $S_{T}$ as a function of $t^{\star}$. The cubic corresponding to these solutions can be expressed in the form

$$
\left(S_{T}-D\right)\left(A S_{T}^{2}+B S_{T}+C\right)=0
$$

with the following coefficients

$$
\begin{aligned}
& A=\nu P_{S} \beta^{2}\left(E\left(t^{\star}\right)-1\right), \\
& B=\nu\left(\left(\left((\nu-1) P_{S}+p_{S}\right) \mu-P_{S} \beta\right) E\left(t^{\star}\right)+\left(2 P_{S} \beta-p_{S} \mu\right)\right), \\
& \left.C=\left(\left(P_{S}+c-p_{S}\right) \nu-c+P_{S} \nu^{2}\right) \mu+c \beta\right) E\left(t^{\star}\right)+\left(p_{S} \mu-P_{S} \beta\right) \nu, \\
& D=\frac{(1-\nu) \mu}{\beta},
\end{aligned}
$$

in which

$$
E\left(t^{\star}\right)=\exp \left[(\beta-\mu(1-\nu))\left(T-t^{\star}\right)\right] .
$$

Thus, one of the solutions of this cubic is $(1-\nu) \mu / \beta$, which corresponds to the endemic equilibrium in absence of control (for $\phi=0)$. Using $S_{T}=(1-\nu) \mu / \beta$ and substituting this solution into Equation (26) yields $S\left(t^{\star}\right)=S_{T}=(1-\nu) \mu / \beta$. Since this line is an equilibrium for the system, it cannot be crossed and thus does not correspond to a switching curve.

In Figure 6, we present numerical simulations of the dynamic optimization problem for different combinations of the parameters $P_{S}$ and $\nu$ and the threshold parameter $R$. In Appendix B, Section 7, an outline of the numerical method employed, the forward backward sweep, is presented. Our numerical simulations indicate that of the remaining two solutions (the roots of a quadratic), only one is biologically relevant. This is the one we use to draw the switching curves in Figure 6 . 
In the special case $P_{S}=0$, the coefficient $A$ equals zero, so $S_{T}=-C / B$. Using Equation (26) yields the switching curve $S^{\star}: t^{\star} \mapsto S\left(t^{\star}\right)$ in the plane $(t, S)$ :

$$
S^{\star}(t)=\frac{\left(\beta c-\mu p_{S} \nu\right) \mathrm{e}^{(T-t)(\beta-\mu(1-\nu))}+\mu(p \nu-c(1-\nu))}{\left(\beta c-\mu p_{S} \nu\right)\left(\mathrm{e}^{(T-t)(\beta-\mu(1-\nu))}-1\right)} .
$$

This is shown in the top and bottom panels of Figure 6 , for the cases $\nu=1$, and $1>\nu>\frac{R}{r}$, respectively.

For $P_{S}>0$, noticing that the coefficient $A$ vanishes with $E(T)=1$, one obtains $S^{\star}(T)=\hat{S}_{T}$ as expressed in equation (25). This means that the switching curve crosses the $t=T$ vertical line at $S=\hat{S}_{T}$. This is shown in the middle panel of Figure 6 provided $\nu P_{S}>c$.

Finally, in Figure 7 we present a graphical depiction of the results of dynamic optimization by dividing the $(R, \nu r)$ plane into distinct regions of differing control strategies as demonstrated by the results of this section.

\section{Discussion and Conclusions}

In this section, we provide both a biological and economic context in which to understand the model considered and the results presented in this paper. There are a number of bio-economical implications that we have gathered from our model and the control problems associated to it. In particular, static optimization, as presented in Section 3.3, yields 3 possible nontrivial strategies, depending on parameter values (see Figure 4): no control (S2), full control (S3), and intermediate control (S4). The region (S2) corresponds to the disease transmission rate being too large with respect to the plant renewal rate or vertical transmission to make the use of cleans seeds viable, which results in the optimal strategy of letting the system approach its endemic equilibrium without any control on the disease. The region (S3) is the opposite; while it is not possible to fully eradicate the disease through clean seeds alone, it is still optimal to use as many clean seeds as possible to minimize the prevalence of the disease. The region (S4) is the case where eradicating the disease is possible by using only a fraction of clean seeds.

Subsidizing clean seeds enables switching from the uncontrolled case (S2) to the controlled cases (S3) or (S4). We derived a very simple bio-economical threshold for the minimal amount of subsidies to make it economically viable for an individual grower to control the disease. However, as subsidizing seeds in this manner only allows us to move along the economical parameter $(r)$ axis in Figure 4, we cannot use subsidies to switch from full control (S3), where the pathogen persists at endemic equilibrium, to intermediate control (S4), where the pathogen goes extinct. The transition from (S3) to (S4) can only be made possible through other possible control methods decreasing horizontal transmission $(R)$, such as introducing partially resistant plants.

Decreasing vertical transmission $(\nu)$ may have the opposite effect as it decreases the utility of clean seeds (Figure 5 and Section 3.4.2). Hence, decreasing vertical transmission can have a counter-productive effect. Breeding for partially resistant plants to decrease the vertical transmission rate of the pathogen $(\nu)$ should be given caution, as it may result in the optimal strategy switching from partial or full control to uncontrolled disease spread. Regions (R3) and (R4) in Figure 5 represent these cases. 


$$
R<1
$$
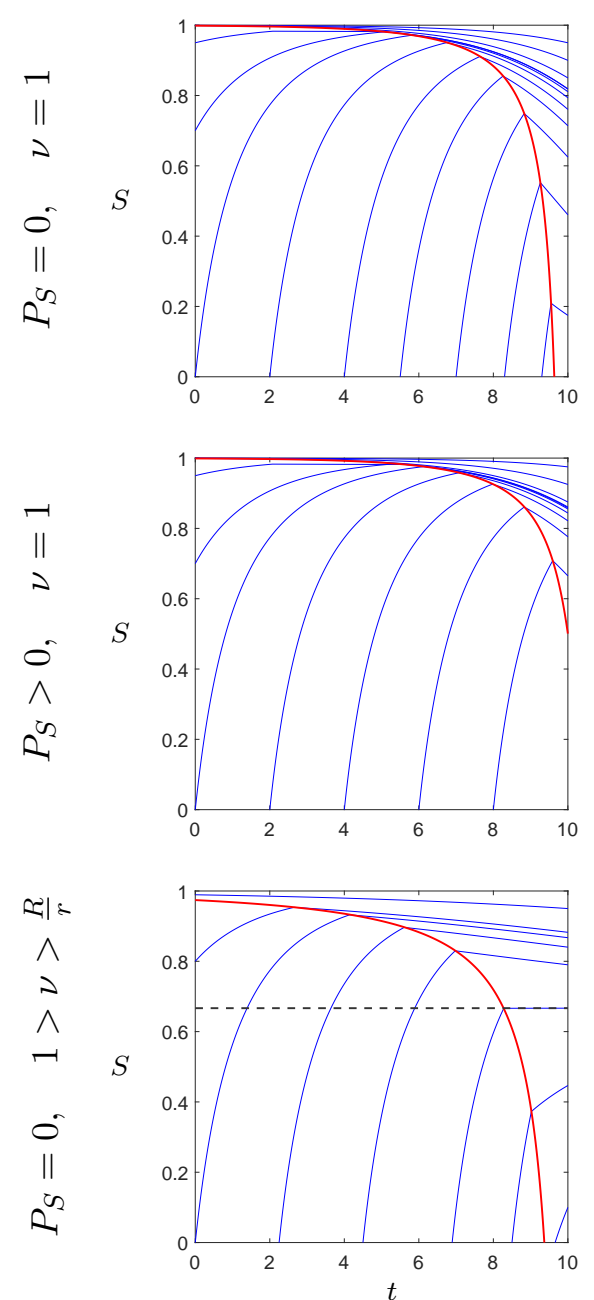

$R>1$
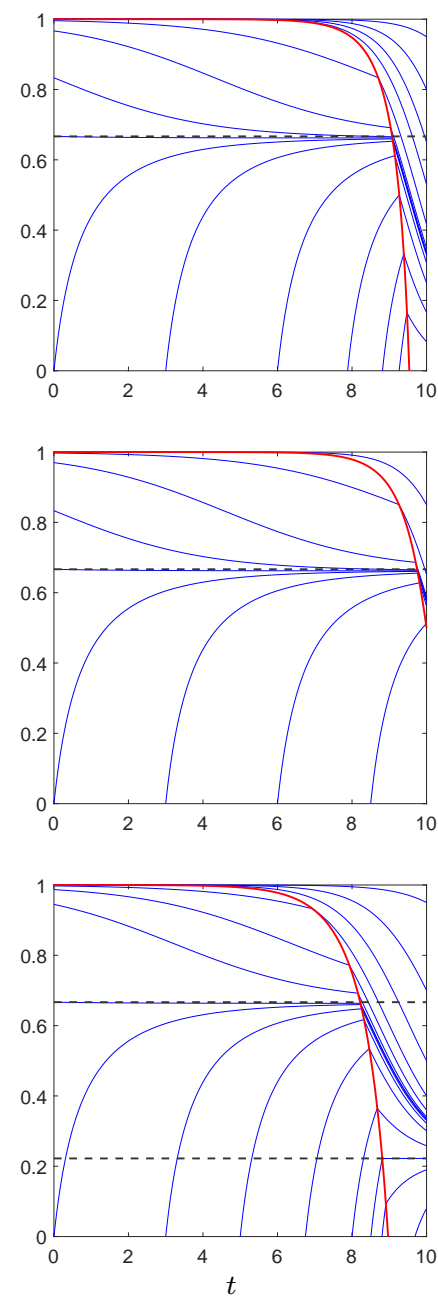

Fig. 6 Dynamics of different combinations of $R, P_{S}$, and $\nu$. Blue curves are trajectories under optimal control, Red curve is the switching curve, and dashed lines are equilibrium values for $\phi=0$ and $\phi=1$ when they differ from $\bar{S}=0$ and $\bar{S}=1$ respectively. (Left Panel) $R<1$ : $\sigma=1, \beta=\frac{1}{2}, p_{S}=3, c=1, \nu \in\left\{1, \frac{2}{3}\right\}, P_{S} \in\{0,2\}$, (Right Panel) $R>1: \sigma=1, \beta=\frac{3}{2}$, $p_{S}=3, c=1, \nu \in\left\{1, \frac{2}{3}\right\}, P_{S} \in\{0,2\}$.

An alternative way to decrease vertical transmission is to sort out diseased seeds from the local pool of seeds to be replanted, which amounts to considering $p>0$ in our model. We assumed $p=0$ for simplicity but our approach can be extended to include positive values of $p$. However, this changes the dynamics and the analysis enough to justify a separate treatment of this case. We therefore leave the $p>0$ case for future research. 


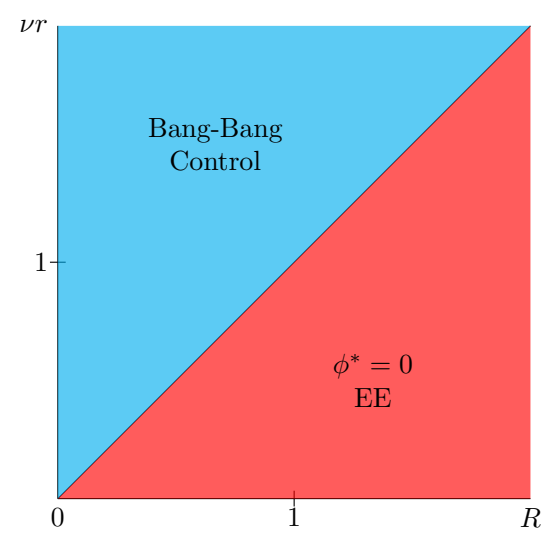

Fig. 7 Results of dynamic optimization: The $(R, \nu r)$ plane is divided into distinct regions with differing control strategies. Examples of this bang-bang control can be seen in Figure 6.

We also developed a dynamic optimization approach maximizing an economically relevant finite-time horizon criterion in Section 4 . This showed that the above distinction between full control (S3) and intermediate control (S4) is likely oversimplified, as in practice it may take a long time to reach an equilibrium. Rather, the optimal strategy is either no control (S2) or bang-bang control, i.e. full control followed by no control (see Figure 7). Intermediate control is not optimal/rational. If a grower uses clean seeds, then they should use only clean seeds (if possible). Although static and dynamic optimization approaches yield qualitatively contrasting results, the subsidies threshold (based on the condition $R<\nu r$ ) is the same following both approaches.

In theory, there may be an incentive to use clean seeds even if the unit cost of clean seeds is greater than the unit benefit of harvested plants, i.e. $r=p_{S} / c<1$. This reflects a population-scale effect in which a few clean seeds help protect a greater number of plants (a phenomenon similar to herd-immunity, as would happen through the use of genetically resistant seeds for instance).

In practice however, the parameter $r$ would unlikely be lower than unity. For instance, consider maize lethal necrosis (MLN) in Kenya. The price of maize is approximately 0.5 USD per kilogram [13]. The average yield is of the order of 2 tons per hectare. The total revenue therefore approximates 1000 USD per hectare $[3,6]$. This is to be compared to the cost of certified seeds, which is approximately 40 USD per hectare [6]. This yields $r \approx 1000 / 40=25$, which is one order of magnitude greater than unity. This makes sense as there are significant implicit costs, including wages and fertilizers, to be covered regardless of clean seed usage [13].

One may finally wonder how $r$ would compare to $R$ (horizontal transmission) and $\nu$ (vertical transmission) in the case of MLN in Kenya. Assuming vertical transmission approximates $5 \%$ [10] yields $r \nu \approx 25 \times 0.05=1.25$. The parameter $R$ can then be estimated as a function of the observed disease prevalence through the relationship $R=(1-\nu) / \bar{I}$. Considering a prevalence of $40 \%$ in absence of control [12] leads to $R \approx 95 / 40=2.375$. Since $R>r \nu$, there would be no economical incentive to use clean seeds to control the disease according to our models. Nevertheless, 
we derived a simple rule for subsidizing clean seeds [6] in this case: namely, the discount on clean seeds should be at least equal to $s \approx 1-1.25 / 2.375 \approx 50 \%$. This way, buying clean seeds to control the disease would become economically viable. Although the above derivation is very rough, such an economical-epidemiological approach may help decision makers define subsidy rates that would be acceptable to informed growers [6].

\section{Appendix A}

We define an extremal trajectory $\hat{\phi}$ as one where the control $\phi$ maximizes or minimizes the Hamiltonian. It is a function of both the state variable(s) (here $S$ ) and the adjoint variable(s) (here $\lambda$ ). We have the following important result [11]:

Theorem 1 (First integral of the energy) If both the dynamics and the running cost are time-invariant, the Hamiltonian is constant along an extremal trajectory.

Proof To avoid any confusion in the notation, we will use Dieudonné's notation for the partial derivatives of the Hamiltonian $\mathcal{H}(S, \lambda, \phi)$,

$$
\begin{aligned}
& \mathrm{D}_{1} \mathcal{H}(S, \lambda, \phi)=-\dot{\lambda}(S, \lambda, \phi), \\
& \mathrm{D}_{2} \mathcal{H}(S, \lambda, \phi)=\dot{S}(S, \lambda, \phi) .
\end{aligned}
$$

Let $\widehat{\mathcal{H}}(S, \lambda):=\mathcal{H}(S, \lambda, \hat{\phi}(S, \lambda))$. It follows from Danskin's theorem (see [8]) that, if the extremalizing $\hat{\phi}$ is unique,

$$
\begin{aligned}
& \mathrm{D}_{1} \widehat{\mathcal{H}}(S, \lambda)=\mathrm{D}_{1} \mathcal{H}(S, \lambda, \hat{\phi}(S, \lambda)), \\
& \mathrm{D}_{2} \widehat{\mathcal{H}}(S, \lambda)=\mathrm{D}_{2} \mathcal{H}(S, \lambda, \hat{\phi}(S, \lambda)) .
\end{aligned}
$$

Moreover, the only way $\hat{\phi}$ could be non-unique would be on a singular arc, where $\mathrm{D}_{3} \mathcal{H}=0$, so that the conclusion of Danskin's theorem would still hold. Hence we have

$$
\frac{\mathrm{d} \widehat{\mathcal{H}}}{\mathrm{d} t}=\mathrm{D}_{1} \widehat{\mathcal{H}}(S, \lambda) \dot{S}(S, \lambda, \hat{\phi})+\mathrm{D}_{2} \widehat{\mathcal{H}}(S, \lambda) \dot{\lambda}(S, \lambda, \hat{\phi})=-\dot{\lambda} \dot{S}+\dot{S} \dot{\lambda}=0
$$

Q.E.D.

\section{Appendix B}

We used two different solution techniques that gave consistent results. In the main text, we analytically solved the optimal control problem in backward time from every possible value of $S_{T}$. Conceptually, this yielded a field of optimal orbits in the plane $(t, S)$ as depicted in Fig. 6. However, the blue curves in Fig. 6 were actually computed using an alternative numerical "forward-backward sweep" method to make sure that the results are correct regardless of the method used. The "forwardbackward sweep" numerical method yielded the same switching curve as predicted from the mathematical analysis. 
In order to numerically compute the optimal control for various values for each parameter, we modified the forward-backward Sweep technique as described in [11]. Our method uses the defined initial conditions of $S(\tau)=S_{\tau}$ and $\lambda(T)=\lambda_{T}$ and an initial guess of the control $\phi(t)=\phi_{0}(t)$ and refines this guess until some convergence criterion has been met. The steps in this method are described as follows:

1. Determine an initial guess for the control $\phi_{0}(t)$.

2. For $i \geq 1$, do the following:

(a) Solve $S(t)$ by moving forward in time on the region $[\tau, T]$ and backward in time on the region $[0, \tau]$ using Runge-Kutta methods (RK4) to approximate the solution to the ODE.

(b) Use these values of of $S(t)$ to approximate the solution of the ODE for $\lambda(t)$ by moving backward in time on the region $[0, T]$ and using RK4 with the same timesteps.

(c) Calculate an approximation to $\psi(t)$ using $S(t)$ and $\lambda(t)$ and use this to determine an update function $\phi_{f i x}(t)$.

(d) Update the guess for $\phi$ using values of $\phi_{i-1}(t)$ and $\phi_{f i x}(t)$. In our model we use $\phi_{i}(t)=(1-\alpha) \phi_{i-1}(t)+\alpha \phi_{f i x}(t)$ for some $\alpha \in(0,1)$.

(e) Continue this process from Step 2 until the following convergence criteria is reached: The relative differences between old and new values, as measured in the infinity norm, are less than a tolerance $(\delta)$ for each of the controls, state variables and the adjoints. If convergence is not reached, go to (a) and repeat, unless a maximum number of iterations have been exceeded.

We used this method to compute the optimal trajectories in Figure 6. In our case, we found that $\alpha=0.1$ was sufficient to generate the optimal control trajectories represented, with $\Delta t=10^{-3}$ the timestep for RK4 and $\phi_{0}(t)=0.5$ the initial guess for the control. We used a tolerance of $\delta=10^{-3}$, for checking the convergence criteria.

\section{Appendix $\mathrm{C}$}

To show that there is no singular region of the optimal control in the cases observed, we turn to proof by contradiction.

Assume $\phi^{*}$ is singular. Then, by the definition of $\phi^{*}$, this means that there exists some interval of time, $I$, such that $\psi(t)=0$ on this interval $I$, we have

$$
\psi(t)=\mu(-c+\lambda(t)(1-S(t)) \nu)=0
$$

Solving for $\lambda$, we get that on $I$,

$$
\lambda(t)=\frac{c}{\nu(1-S(t))},
$$

whenever $S(t) \neq 1$. We can then take the time derivative of this quantity and the state equation (5) to get the relationship

$$
\frac{\mathrm{d} \lambda}{\mathrm{d} t}=\frac{c}{\nu(1-S(t))}(-\beta S(t)+\mu \phi \nu+\mu(1-\nu)) .
$$


Equating the above to the relationship derived from the Hamiltonian in Equation (24) and cancelling out terms, we get the identity

$$
-\mu p_{S}+\frac{c}{\nu} \beta=0
$$

Using the definitions of $R$ and $r$, we can show that the above identity is equivalent to $R=r \nu$, which is the line dividing the regions in Figure 7 . Since we are only considering the cases $R>r \nu$ and $R<r \nu$, (i.e. $R \neq r \nu$ ), we conclude that there is no singular region of the optimal control along the switching curve.

\section{Appendix D}

In the case of an infinite horizon, we are interested in maximizing

$$
J(\phi(\cdot))=\mu \int_{0}^{\infty} e^{-\delta t}\left(p_{S} S(t)-c \phi(t)\right) \mathrm{d} t,
$$

with respect to $\phi \in[0,1]$, subject to the state equation (5) again. In the above $e^{-\delta t}$ is a discount term, with the parameter $\delta>0$.

To show that the integral in Equation (30) converges, we use the property that both $S(t)$ and $\phi(t)$ are bounded between 0 and 1 :

$$
\begin{aligned}
\left|\mu \int_{0}^{\infty} e^{-\delta t}\left(p_{S} S(t)-c \phi(t)\right) \mathrm{d} t\right| & \leq \mu \int_{0}^{\infty}\left|e^{-\delta t}\left(p_{S} S(t)-c \phi(t)\right)\right| \mathrm{d} t \\
& \leq \mu \int_{0}^{\infty} e^{-\delta t} \max \left(p_{S}, c\right) \mathrm{d} t=\frac{\mu \max \left(p_{S}, c\right)}{\delta} .
\end{aligned}
$$

The Hamiltonian $\mathcal{H}$ and the switch function $\psi(t)$ are slightly modified with respect to the finite-horizon problem addressed in Section 4. This results in the adjoint equation

$$
\frac{d \lambda}{d t}=-\mu p_{S} e^{-\delta t}+\lambda(t)[(1-S(t)) \beta+(\nu \mu \phi(t)-\beta S(t)+\mu(1-\nu))],
$$

and switch function

$$
\psi(t)=\mu\left(-c e^{-\delta t}+\lambda(t)(1-S(t)) \nu\right)
$$

We are interested in a singular control $\phi^{*} \in(0,1)$ such that $\psi=0$ along the singular part of the optimal trajectory. This yields

$$
\lambda(t)=\frac{c e^{-\delta t}}{\nu(1-S(t))},
$$

and differentiating this in respect to time gives us

$$
\frac{\mathrm{d} \lambda}{\mathrm{d} t}=c e^{-\delta t} \frac{-\delta+\mu \phi \nu-\beta S+\mu(1-\nu)}{\nu(1-S(t))} .
$$


Setting these two equations for the time derivative of $\lambda$ equal to each other and solving for $S(t)$ results in the following equation for $S^{*}$

$$
S^{*}=1-\frac{\delta}{\nu \mu\left(r-\frac{R}{\nu}\right)} .
$$

A necessary condition for the singular control to exist is $S^{*}<1$, which occurs whenever $R<r \nu$. This ratio has appeared in previous analysis, and represents the conditions where it is economically beneficial to use clean seeds. From here, we have that $S^{*}>0$ if and only if

$$
\delta<\nu \mu\left(r-\frac{R}{\nu}\right)
$$

meaning the discount rate must be small enough for long term interests to prevail over short term interests.

If $\phi^{*} \in(0,1)$ is constant, then the dynamics of the system result in the equilibrium point

$$
\bar{S}=\frac{1}{R}\left(\phi^{*} \nu+(1-\nu)\right) .
$$

Equating $\bar{S}$ and $S^{*}$ results in the following expression for $\phi^{*}$

$$
\phi^{*}=\frac{1}{\nu}\left(R\left(1-\frac{\delta}{\nu \mu\left(r-\frac{R}{\nu}\right)}\right)-(1-\nu)\right)=\frac{1}{\nu}\left(R S^{*}-(1-\nu)\right) .
$$

Letting $K=\nu \mu(r-R / \nu)$, we get that $0<\phi^{*}<1$ only if

$$
\max \left(K\left(1-\frac{1}{R}\right), 0\right)<\delta<K\left(1-\frac{1-\nu}{R}\right),
$$

which requires $\mathcal{R}_{0}=R+\nu>1$ otherwise the upper bound is negative.

Acknowledgements This material is based upon research supported by the Thomas Jefferson Fund (TJF) of the Embassy of France in the United States, and the FACE Foundation. The authors thank anonymous reviewers for their helpful suggestions.

\section{Conflict of interest}

The authors declare that they have no conflicts of interest.

\section{References}

1. Alabi, O., Rayapati, N., Kumar, L.: Cassava mosaic disease: A curse to food security in Sub-Saharan Africa. APSnet Features (2011). DOI 10.1094/APSnetFeature-2011-0701

2. Andersen, K., Buddenhagen, C., Rachkara, P., Gibson, R., Kalule, S., Phillips, D., Garrett, K.: Modeling epidemics in seed systems and landscapes to guide management strategies: The case of sweet potato in Northern Uganda. Phytopathology 109(9), 1519-1532 (2019)

3. Beyene, Y., Gowda, M., Suresh, L., Mugo, S., Olsen, M., Oikeh, S.O., Juma, C., Tarekegne, A., Prasanna, B.M.: Genetic analysis of tropical maize inbred lines for resistance to maize lethal necrosis disease. Euphytica 213(9), 224 (2017) 
4. Bokil, V., Allen, L., Jeger, M., Lenhart, S.: Optimal control of a vectored plant disease model for a crop with continuous replanting. Journal of Biological Dynamics 13, 1-29 (2019)

5. van den Bosch, F., Jeger, M., Gilligan, C.: Disease control and its selection for damaging plant virus strains in vegetatively propagated staple food crops; a theoretical assessment. Proceedings of the Royal Society B: Biological Sciences 274(1606), 11-18 (2006)

6. Bulte, E., Cecchi, F., Lensink, R., Marr, A., Van Asseldonk, M.: Does bundling crop insurance with certified seeds crowd-in investments? Experimental evidence from Kenya. Journal of Economic Behavior \& Organization (2019)

7. Bussell, E., Dangerfield, C., Gilligan, C., Cunniffe, N.: Applying optimal control theory to complex epidemiological models to inform real-world disease management. Philosophical Transactions of the Royal Society B: Biological Sciences 374, 20180284 (2019)

8. Danskin, J.M.: The Theory of Max Min. Springer, Berlin (1967)

9. Ferris, A.C., Stutt, R.O., Godding, D., Gilligan, C.A.: Computational models to improve surveillance for cassava brown streak disease and minimize yield loss. PLoS computational biology 16(7), e1007823 (2020)

10. Hilker, F.M., Allen, L.J., Bokil, V.A., Briggs, C.J., Feng, Z., Garrett, K.A., Gross, L.J., Hamelin, F.M., Jeger, M.J., Manore, C.A., et al.: Modeling virus coinfection to inform management of maize lethal necrosis in kenya. Phytopathology 107(10), 1095-1108 (2017)

11. Lenhart, S., Workman, J.: Optimal Control Applied to Biological Models. CRC Press (2007)

12. Mahuku, G., Lockhart, B.E., Wanjala, B., Jones, M.W., Kimunye, J.N., Stewart, L.R., Cassone, B.J., Sevgan, S., Nyasani, J.O., Kusia, E., Kumar, P.L., Niblett, C.L., Kiggundu, A., Asea, G., Pappu, H.R., Wangai, A., Prasanna, B.M., Redinbaugh, M.G.: Maize lethal necrosis (MLN), an emerging threat to maize-based food security in Sub-Saharan Africa. Phytopathology 105(7), 956-965 (2015)

13. Marenya, P.P., Erenstein, O., Prasanna, B., Makumbi, D., Jumbo, M., Beyene, Y.: Maize lethal necrosis disease: evaluating agronomic and genetic control strategies for Ethiopia and Kenya. Agricultural Systems 162, 220-228 (2018)

14. McQuaid, C., Sseruwagi, P., Pariyo, A., Van den Bosch, F.: Cassava brown streak disease and the sustainability of a clean seed system. Plant pathology 65(2), 299-309 (2016)

15. McQuaid, C.F., van den Bosch, F., Szyniszewska, A., Alicai, T., Pariyo, A., Chikoti, P.C., Gilligan, C.A.: Spatial dynamics and control of a crop pathogen with mixed-mode transmission. PLoS computational biology 13(7), e1005654 (2017)

16. Redinbaugh, M.G., Stewart, L.R.: Maize lethal necrosis: An emerging, synergistic viral disease. Annual Review of Virology 5, 301-322 (2018)

17. Rey, C., Vanderschuren, H.: Cassava mosaic and brown streak diseases: current perspectives and beyond. Annual review of virology 4, 429-452 (2017)

18. Thomas-Sharma, S., Abdurahman, A., Ali, S., Andrade-Piedra, J., Bao, S., Charkowski, A., Crook, D., Kadian, M., Kromann, P., Struik, P.C., et al.: Seed degeneration in potato: the need for an integrated seed health strategy to mitigate the problem in developing countries. Plant Pathology 65(1), 3-16 (2016)

19. Thomas-Sharma, S., Andrade-Piedra, J., Carvajal Yepes, M., Hernandez Nopsa, J., Jeger, M., Jones, R., Kromann, P., Legg, J., Yuen, J., Forbes, G., et al.: A risk assessment framework for seed degeneration: Informing an integrated seed health strategy for vegetatively propagated crops. Phytopathology 107(10), 1123-1135 (2017) 